\title{
Special issue on learning and intelligent optimization
}

\author{
Roberto Battiti • Bart Selman • Thomas Stützle
}

Published online: 15 April 2011

(C) Springer Science+Business Media B.V. 2011

Learning and optimization are often treated as two different research fields. However, it is clear that human problem solving strongly relies on learning in various forms. For sure, learning takes place when facing an initially unknown problem. In addition, while trying to solve it, by learning one obtains insights into its structure and derives improved strategies for solving it. Clearly, previous experience with problem solving also helps us humans to solve newly arising problems with increased efficiency. When seen from this perspective, learning and solving optimization problems are two closely related fields. The LION conference series on Learning and Intelligent Optimization makes this link explicit by declaring it its main theme.

This special issue collects the extended versions of papers that have been presented at the second and the third LION conferences and a number of additional papers that have been contributed by authors active in this interdisciplinary research subject. Of the sixteen submitted papers, six have been accepted for publication.

The first paper by Olivier Caelen and Gianluca Bontempi on A Dynamic Programming Strategy to Balance Exploration and Exploitation in the Bandit Problem introduces a new algorithm that is based on dynamic programming and on estimation techniques to tackle the well-known $K$-armed bandit problem. The following article, Analyzing Bandit-based Adaptive Operator Selection Mechanisms by Álvaro Fialho, Luis Da Costa, Marc Schoenauer and Michèle Sebag, studies how the multi-armed

\footnotetext{
R. Battiti

Dipartimento di Ingegneria e Scienza dell'Informazione, Università degli Studi di Trento, Trento, Italy e-mail: battiti@disi.unitn.it

B. Selman

Department of Computer Science, Cornell University, Ithaca, NY, USA e-mail: selman@cs.cornell.edu

T. Stützle $(\varangle)$

IRIDIA, CoDE, Université Libre de Bruxelles (ULB), Brussels, Belgium e-mail: stuetzle@ulb.ac.be
} 
bandit framework can be exploited to adapt the probabilities of choosing specific operators at algorithm run-time with the goal of improving performance. Frank Hutter, Holger H. Hoos and Kevin Leyton-Brown study Tradeoffs in the Empirical Evaluation of Competing Algorithm Designs. This study is based on an empirical analysis approach that characterizes tradeoffs between the number of algorithm designs examined, the number of problem instances tested, and the stopping time for each algorithm run; these tradeoffs are important to consider when using automated methods for offline algorithm configuration. The paper Learning Cluster-based Structure to Solve Constraint Satisfaction Problems by Xingjian Li and Susan L. Epstein shows that the extraction of substructures through local search and the exploitation of the knowledge about these substructures leads to considerable performance gains for complete search algorithms for constraint satisfaction problems. The goal of the work by Vasileios Vasilikos and Michail G. Lagoudakis, which is titled Optimization of Heuristic Search using Recursive Algorithm Selection and Reinforcement Learning, is also to improve the performance of complete search algorithms. These goals are here reached by using reinforcement learning to learn at each recursive call in a tree search, which of various possible strategies to follow. Finally, the paper Genetic Algorithms and Particle Swarm Optimization for Exploratory Projection Pursuit by Alain Berro, Souad Larabi Marie-Sainte and Anne Ruiz-Gazen explores the usage of genetic algorithms to optimize indices that define projections of high-dimensional data into a low-dimensional space, a procedure that is useful for exploratory data analysis.

We would like to thank all authors who have submitted their articles and the referees for their detailed and insightful comments. We hope that this special issue will be useful as an example of the exiting work that is developed in the intersection between machine learning and applied optimization and that the articles contained in it will inspire more research efforts in this direction.

Roberto Battiti, Bart Selman \& Thomas Stützle December 2010 